\title{
Editorial
}

\section{Accept and Respect the Uniqueness of Oral Cancer}

Oral cancer has become a major cause of concern worldwide due to its increasingly growing incidence, late diagnosis, patient's inferior quality of life and poor prognosis. Apart from the traditional treatment modalities like radiotherapy and chemotherapy, many newer therapies (targeted drug therapy, immunotherapy, etc.) are under extensive research with varying degree of success rates. Moreover, thousands of newer compounds are under preclinical and clinical trials, the fate of which is still unpredictable.

To win any war, it is important to know the enemy inside out. This appears to be apt with oral cancer as it displays diversified uniqueness, which one should know before developing targeted therapeutic strategies.

The most unique aspect of oral cancer is its association with numerous etiological agents. These include tobacco consumption (chewing, smoking and snuff), alcohol, betel nut, human papillomavirus (HPV) (16 and 18), chronic trauma, Candida, chronic inflammation, inherited genetic mutations and so on. No other malignancy of the body has such a vast array of etiological agents. Each one has its own unique process of carcinogenesis causing upregulation or downregulation of specific sets of signaling pathways responsible for cell migration, invasion, proliferation and survival. In our personal experience, oral cancer patients usually have varied combinations of aforementioned habits, making carcinogenesis a more complex process. In fact, each tobacco consumption-related habit (chewing, smoking, snuff, etc.) has unique mechanism of carcinogenesis. This 'varied mechanisms of carcinogenesis'-related aspect is responsible for the intertumoral or intratumoral heterogeneity at molecular level, which is one of the causes for cancer drug resistance. Especially in oral cancer, this will be one of the biggest challenges for tomorrow's 'personalized cancer medicine'.

Another factor responsible for uniqueness of oral cancer is its association with HPV (16 and 18) virus. A very few other cancers like cervical cancer shows an association with this virus. The mechanism of carcinogenesis in oral cancer is same as that of other cancers of the body. However, its association with other etiological agents (tobacco, alcohol, betal nut, inflammation, etc.) makes HPV-associated carcinogenesis unique in case of oral cancer. HPV 16 and 18 oncoproteins also interact with different signaling molecules, thus altering the signaling pathways responsible for carcinogenesis, the aspect that must be kept in mind while developing targeted therapy. ${ }^{1}$ The HPV-associated oral cancer mainly affects oropharyngeal area and has better prognosis than non-HPV-associated oral cancer. Hence, we believe that they should be considered as a separate entity for studying the targeted drug response.

In addition to this what makes oral cancer unique is attributed to its association with different oral potentially malignant disorders (OPMDs). According to the recent classification of OPMDs, there are almost 27 disorders, which can be associated with oral cancer. ${ }^{2}$ We believe that no other cancer of the body has so many diversified precursors in the form of OPMDs. Each disorder carries its own mechanism of carcinogenesis during malignant transformation, making each oral squamous cell carcinoma (OSCC) patient unique at the molecular level. ${ }^{3}$ In this context, it has been observed that oral submucous fibrosis (OSMF) associated with OSCC shows better differentiation of tumor cells and better prognosis when compared with non-OSMF-associated OSCC. ${ }^{4}$

It is known that local environment is responsible for alteration in the disease process. Presence of microbial flora with wide variety of microorganisms makes oral cavity unique. Our personal observation shows that virtually all the oral cancer cases are associated with secondary infection derived from oral microbial flora. We believe that this secondary infection/inflammation can evoke 'inflammation-associated' mutational changes in the already mutated oral cancer cells, thus fueling the already present tumor heterogeneity and cancer drug resistance. In this regard, therapeutic opportunities associated with 'inflammation-mediated carcinogenesis' have promising future, especially in case of oral cancer. ${ }^{5}$

Recently, we have proposed that oral cancer is unique in terms of chemotherapeutic and targeted drug metabolism. ${ }^{6}$ Factors such as unique microenvironment, poor oral hygiene, OPMDs, tumor-associated inflammation and age could act additively in elevating the levels of circulating interleukin-6 and C-reactive protein. This could lead to transcriptional suppression of CYP3A4 function and subsequently to metabolism of chemotherapeutic drugs. Thus, OSCC, in terms of metabolism of chemotherapeutic drugs and its responsiveness, is different from other carcinomas of the body and warrants special therapeutic attention. In comparison with the internal malignancies, oral cancer is characterized by easy clinical visibility and detection. Even though oral cancer is diagnosed in late stages due to delay on patient's part (appraisal delay 
+ illness delay + utilization delay) self-mouth examination is easier to implement by health education than screening by health workers. It empowers the individual to maintain his/her own oral health and thus could facilitate early detection of oral cancer. ${ }^{7}$ In our opinion, this could be a promising method for early detection of reducing the morbidity and mortality attributed to oral cancer.

\section{REFERENCES}

1. Sarode GS, Sarode SC. E6 oncoprotein interaction with paxillin and FAK. Oral Oncol 2014 Apr;50(4):e17.

2. Sarode SC, Sarode GS, Karmarkar S, Tupkari JV. A new classification for potentially malignant disorders of the oral cavity. Oral Oncol 2011 Sep;47(9):920-921.

3. Sarode SC, Sarode GS, Tupkari JV. Oral potentially malignant disorders: precising the definition. Oral Oncol 2012 Sep;48(9):759-760.

4. Sarode SC, Sarode GS. Better grade of tumor differentiation of oral squamous cell carcinoma arising in background of oral submucous fibrosis. Med Hypotheses 2013 Oct; 81(4):540-543.

5. Sarode SC, Sarode GS, Patil A. Therapeutic aspects of the inflammation mediated oral carcinogenesis. Oral Oncol 2014 Apr;50(4):e13-e14.

6. Sarode SC, Sarode GS. Is oral cancer unique in terms of chemotherapeutic and targeted drug metabolism? J Oral Maxillofac Surg 2015 Jan;73(1):4-6.

7. Sarode SC, Sarode GS, Karmarkar S. Early detection of oral cancer: detector lies within. Oral Oncol 2012 Mar;48(3):193-194.

Gargi S Sarode MDS

Associate Professor

Department of Oral Pathology and Microbiology

DY Patil Dental College and Hospital

Dr DY Patil Vidyapeeth, Sant-Tukaramnagar, Pimpri,

Pune-18, Maharashtra, India, e-mail: gargi14@gmail.com

Sachin C Sarode MDS

Professor

Department of Oral Pathology and Microbiology

Dr DY Patil Dental College and Hospital

Dr DY Patil Vidyapeeth, Sant-Tukaramnagar, Pimpri

Pune-18, Maharashtra, India

Phone: +919922491465

e-mail: drsachinsarode@gmail.com

Shankargouda Patil

Associate Professor, Department of Oral Pathology,

Faculty of Dental Sciences

MS Ramaiah University of Applied Sciences

Bengaluru, Karnataka, India e-mail: sbpatil1612@gmail.com 